\title{
When univariate model-free time series prediction is better than multivariate?
}

\author{
Masayoshi Chayama ${ }^{\mathrm{a}, *}$, Yoshito Hirata ${ }^{\mathrm{a}, \mathrm{b}}$ \\ ${ }^{a}$ Graduate School of Mathematical Informatics, The University of Tokyo, Tokyo \\ 113-8656, Japan. \\ ${ }^{b}$ Institute of Industrial Science, The University of Tokyo, Tokyo 153-8505, Japan.
}

\begin{abstract}
The delay coordinate method is known to be a practically useful technique for reconstructing the states of an observed system. While this method is theoretically supported by Takens' embedding theorem concerning observations of a scalar time series, we can extend the method to include a multivariate time series. It is often assumed that a better prediction can be obtained using a multivariate time series than by using a scalar time series. However, multivariate time series contains various types of information, and it may be difficult to extract information that is useful for predicting the states. Thus, univariate prediction may sometimes be superior to multivariate prediction. Here, we compare univariate model-free time series predictions with multivariate ones, and demonstrate that univariate model-free prediction is better than multivariate one when the prediction steps are small, while multivariate prediction performs better when the prediction steps become larger. We show the validity of the former finding by using artificial datasets generated from the Lorenz 96 models and a real solar irradiance dataset. The results indicate that it is possible to determine which method is the best choice by considering how far into the future we want to predict.
\end{abstract}

Keywords: delay coordinates, solar irradiance, time series prediction, multivariate prediction

*tea-mountain@sat.t.u-tokyo.ac.jp

Preprint submitted to Physical Letter A

May 13, 2016 


\section{Introduction}

Embedding methods are employed to reconstruct attractors from a time series. In particular, they are often used for making predictions for a time series over a short period. It is known that a chaotic dynamical system can be reconstructed from a scalar time series of observations of the state of a dynamical system under the condition stated in Takens' theorem [1, 2], which is that the embedding dimension is larger than twice the dimension for the underlying dynamics. It is commonly assumed that a multivariate time series can provide a better future prediction than a scalar time series [3]. For example, weather forecasts are based on many types of data that are observed in various locations. Actually, if a distance between 2 points becomes smaller, a correlation coefficient of time series data of solar irradiance becomes higher [4]. Therefore, we commonly presume that we can obtain a better prediction by using a multivariate time series than by using a scalar time series. However, multivariate time series contains various types of information, and it is difficult to extract information that is useful for predicting the states [5]. Therefore, we investigate the conditions under which univariate time series prediction is superior to multivariate time series prediction. We use model-free prediction based on embedding techniques $[6,7,5]$. There are some existing methods for predicting a multivariate time series. In particular, those described in $[8,9,10]$ adopt a linear approach. In this paper, we compare multivariate predictions with univariate predictions by using a nonlinear approach.

The remainder of this paper is organized as follows. In Section 2, we discuss the theoretical framework of delay coordinates and Takens' embedding theorem. In addition, we discuss our prediction methods, which are based on the analogue method. In Section 3, we compare our predictions on artificial datasets generated from the Lorenz 96 models [11] and a real dataset of solar irradiance. In Section 4, we discuss our results and conclude the paper. 


\section{Material and methods}

\subsection{Takens' embedding theorem}

Takens' embedding theorem $[1,2]$ provides information regarding the hidden status of a dynamical system. Using this theorem, we can reconstruct its attractor from a limited number of observations. This theorem can be applied in the domain of time series predictions.

First, we consider a dynamical system in which Takens' embedding theorem can be applied. Suppose that we have a dynamical system $x(t) \in \mathbb{R}^{m}$ and an observational time series $s(t) \in \mathbb{R}$ given as follows:

$$
\begin{aligned}
\frac{d x(t)}{d t} & =f(x(t)), \\
s(t) & =g(x(t)),
\end{aligned}
$$

where $f: \mathbb{R}^{m} \rightarrow \mathbb{R}^{m}$ represents the underlying dynamics, $g: \mathbb{R}^{m} \rightarrow \mathbb{R}$ is an observation function, and $T \in \mathbb{R}$ denotes a time delay. Then, we define a function $G_{d, T}: \mathbb{R}^{m} \rightarrow \mathbb{R}^{d}$ for obtaining delay coordinates as follows:

$$
\begin{aligned}
& G_{d, T}(x(t-(d-1) T)) \\
= & (s(t-(d-1) T), s(t-(d-2) T), \ldots, s(t-T), s(t)),
\end{aligned}
$$

where $d \in \mathbb{N}$ represents the embedding dimension. Takens demonstrated that if $d \geq 2 m+1$, then it holds that $G_{d, T}$ and the derivative of $G_{d, T}$ are one-to-one, namely, the maps are injective on the attractor of $f$.

\subsection{Analogue method}

The analogue method was first proposed by Lorenz [6]. We define a set $N_{d, T}(t)$ of indices for the $K$ nearest neighbors at a time $t$. Then, a direct prediction for $p$ steps in the future is formulated as follows:

$$
\begin{aligned}
\hat{s}(t+p) & =\frac{1}{\left|N_{d, T}(t)\right|} \sum_{l \in N_{d, T}(t)} s(l+p) \\
& =h\left(G_{d, T}(x(t-(d-1) T)), p\right),
\end{aligned}
$$

where $p$ denotes the number of prediction steps. Furthermore, we consider an iterative prediction. In the iterative prediction, we use one step prediction $\hat{s}(t+1)$ and feed it as an input repeatedly $p$ times to generate $p$ steps ahead prediction. 


\subsection{Multivariate method}

In this paper, we consider a multivariate version of prediction. We suppose that a multidimensional dynamical system $\mathbf{x}(t)$ and an observed time series $\mathbf{s}(t)$ are defined by

$$
\begin{aligned}
\mathbf{x}(t) & =\left(x_{1}(t), x_{2}(t), \ldots, x_{m}(t)\right) \in \mathbb{R}^{m} \\
\mathbf{s}(t) & =\left(s_{m_{1}}(t), s_{m_{2}}(t), \ldots, s_{m_{I}}(t)\right) \in \mathbb{R}^{I} .
\end{aligned}
$$

In particular, these are given as

$$
\begin{aligned}
\frac{d \mathbf{x}(t)}{d t} & =f(\mathbf{x}(t)), \\
\mathbf{s}(t) & =g(\mathbf{x}(t)),
\end{aligned}
$$

where $f: \mathbb{R}^{m} \rightarrow \mathbb{R}^{m}$ represents the underlying dynamics, and $g: \mathbb{R}^{m} \rightarrow \mathbb{R}^{I}$ is an observation function. Now, we define a different version of the function to obtain delay coordinates. By following $[2,12]$, let $G_{d, T}: \mathbb{R}^{m} \rightarrow \mathbb{R}^{I d}$ be a function defined as follows:

$$
\begin{aligned}
& G_{d, T}(\mathbf{x}(t-(d-1) T)) \\
= & \left(s_{1}(t-(d-1) T), s_{1}(t-(d-2) T), \ldots, s_{1}(t-T), s_{1}(t),\right. \\
& s_{2}(t-(d-1) T), s_{2}(t-(d-2) T), \ldots, s_{2}(t-T), s_{2}(t), \\
& \ldots, \\
& \left.s_{I}(t-(d-1) T), s_{I}(t-(d-2) T), \ldots, s_{I}(t-T), s_{I}(t)\right),
\end{aligned}
$$

where $T \in \mathbb{R}$ represents a time delay and $d \in \mathbb{R}$ a parameter for a delay dimension. According to [13], if $d I \geq 2 m+1$, then the generic property holds that $G_{d, T}$ is one-to-one, and the derivative of $G_{d, T}$ is also one-to-one on the attractor of $f$. We define a set $N_{I, d, T}(t)$ of $K$ nearest neighbors for a time $t$. Then, a prediction for $p$ steps into the future is given as follows:

$$
\hat{\mathbf{s}}(t+p)=\frac{1}{\left|N_{I, d, T}(t)\right|} \sum_{l \in N_{I, d, T}(t)} \mathbf{s}(l+p),
$$

where $p$ denotes the number of prediction steps. 


\section{Results and discussion}

\subsection{Lorenz 96 models}

In this section, we present some results from numerical experiments by using artificial data. We compare univariate time series predictions with multivariate time series predictions by using five types of time series data. Dataset (i) was constructed from the Lorenz 96 I model [11] without observational noise, while dataset (ii) was constructed from the same model and contains observational noise, which was generated by $10 \%$ white noise. This value means the ratio between the standard deviations of noise-free signal and noise. In addition, dataset (iii) was constructed from the same model and contains dynamical noise. Datasets (iv) and (v) were constructed from the Lorenz 96 II model [11], and the latter contains observational noise generated by $10 \%$ white noise. The equations of the Lorenz 96 I model are given as follows:

$$
\frac{d x_{i}}{d t}=x_{i-1}\left(x_{i+1}-x_{i-2}\right)-x_{i}+F
$$

for $i=1,2, \ldots, n$, where the index $i$ is cyclic. We set $F=8$ and $n=10$. In addition, the time step for integrating differential equations is fixed as $d t=0.02$ if not mentioned. We observe $x_{i}(i=1,2, \ldots, n)$ to generate a time series. A given time series consists of the coordinate $x_{i}(t)$ with $i$ fixed. We set $T=0.02$ except for the discussion in Section 4.2. The equations of the Lorenz 96 II model are given as follows:

$$
\begin{aligned}
\frac{d x_{i}}{d t} & =x_{i-1}\left(x_{i+1}-x_{i-2}\right)-x_{i}+F-\frac{h c}{b} \sum_{j=1}^{m} y_{i, j} \\
\frac{d y_{i, j}}{d t} & =c b y_{i, j+1}\left(y_{i, j-1}-y_{i, j+2}\right)-c y_{i, j}+\frac{h c}{b} x_{i}
\end{aligned}
$$

for $i=1,2, \ldots, n$ and $j=1,2, \ldots, m$, where the index $i$ and $j$ are cyclic. We set $n=8$ and $m=4$. In addition, the time step for integrating differential equations is fixed as $d t=0.002$. Moreover, the coefficients are set to $h=1$ and $b=c=10$. We observe $y_{i, j}(i=1,2, \ldots, n, j=1,2, \ldots, m)$ to generate a time series. A given time series consists of the coordinate $y_{i j}(t)$ with $i$ and $j$ fixed as well as $x_{i}$ for $i$ fixed. We set $T=0.002$. The dataset which 
contains dynamical noise are calculated by solving some stochastic differential equations. We calculate approximate solutions by the Euler-Maruyama method [14]. Dataset (iii) is given by the following equations:

$$
\begin{aligned}
x_{i+1}(t+d t) \approx & x_{i}(t)+d t\left(x_{i-1}(t)\left(x_{i+1}(t)-x_{i-2}(t)\right)-x_{i}(t)+F\right) \\
& +\sqrt{d t} z_{i}(t), \\
z_{i}(t) \sim & N\left(0, \sigma_{i}^{2}\right),
\end{aligned}
$$

where $z_{i}(t)$ means a Gaussian random variable of mean 0 and standard deviation $\sigma_{i}$ for the $i$ th point at time $t$.

\subsubsection{Univariate prediction vs. multivariate prediction}

For the first numerical experiment, we used datasets (i) and (iv). We set the length of the artificial time series to 40000, and predicted the next 10000 points. Figure 1 presents the prediction results for (i) and (iv), for univariate and multivariate predictions. For dataset (i), both of methods provide the prediction of $x_{4}$. The univariate prediction is generated from $x_{4}$, and the multivariate prediction is generated from $x_{3}, x_{4}$, and $x_{5}$. For dataset (iv), both of methods provide the prediction of $y_{1,1}$. The univariate prediction is generated from $y_{1,1}$, and the multivariate prediction is generated from $x_{8}, y_{1,1}$, and $y_{1,2}$. The type of used prediction method is the direct prediction. In our numerical experiments, the number of neighboring points $\left|N_{I, d, T}(t)\right|$ is $K$. For comparison, we present the mean prediction error and the persistence prediction error. In the mean prediction, we let a mean value of time series data be a prediction for the future values. In the persistence prediction, we declare a current value as a prediction for the future values.

Because both univariate and multivariate prediction perform better than the mean and persistence predictions, we conclude that these methods generally provide good prediction results. In calculating distances of nearest neighbors $N_{I, d, T}(t)$, we use the Euclidean norm because the Euclidean norm is a simple and common norm. For a smaller number of steps, univariate prediction performed better than multivariate prediction. However, as the number of steps increased, this relationship was reversed. We hypothesize that because multivaraite prediction has access with more variables, multivariate prediction would contain more information, which has a negative influence on the prediction accuracy for a small number of steps [5]. In particular, this influence is considered to be caused by items of information that have low correlations with the observable that we predict. According to Fig. 1, it 
seems to be impossible to decide which prediction method is more accurate because both of the prediction results have large standard deviations. Thus, we used the t-test and Wilcoxon rank sum test to investigate whether or not we have a significant difference in two one-step ahead prediction results. Then, both tests confirmed that there is a significant difference between the means and medians of univariate and multivariate predictions, respectively. The p-values of the t-test and the Wilcoxon rank sum test were both less than 0.0001, indicating the rejections of the null hypothesis of equal mean$\mathrm{s}$ and medians, respectively, at the default 5\% significance level. Hence, univariate prediction is more useful than multivariate prediction for shortterm predictions. However, multivariate prediction is more accurate than univariate prediction for a longer-term prediction.
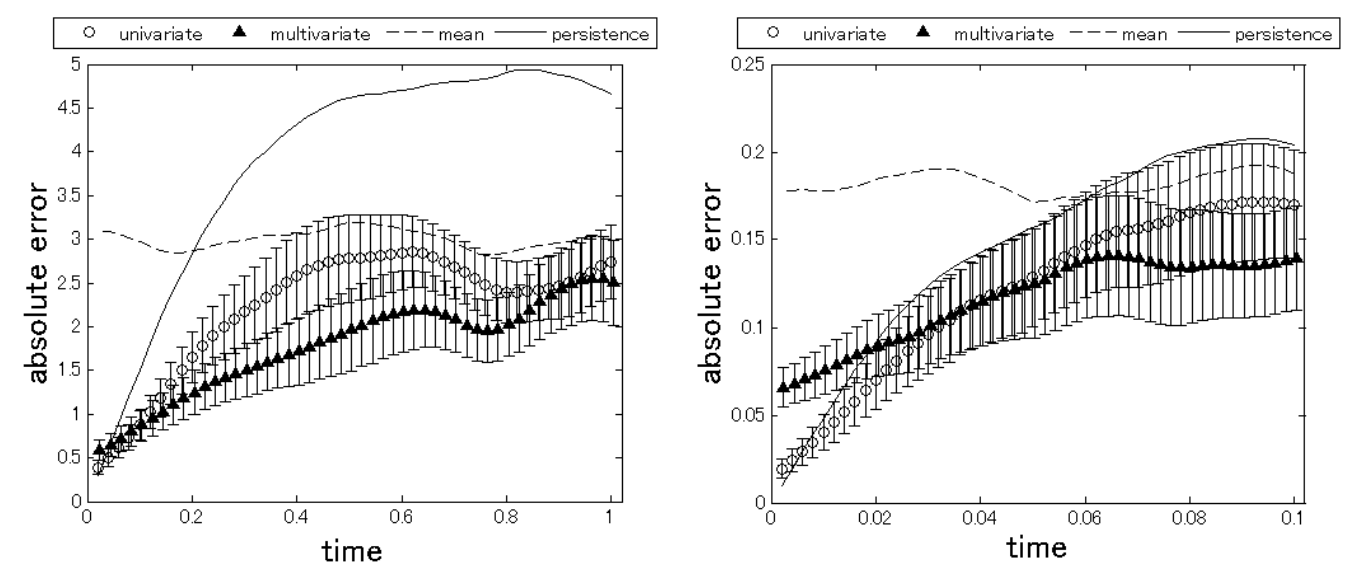

Figure 1: Prediction results for time series data for the Lorenz 96 I (left) and II (right) model. The methods applied are univariate and multivariate predictions. An average was taken over 100 runs for each step. We fixed $d=30$. The number of neighboring points is $K=10$. The scale of the error bar is $20 \%$ of the standard deviation.

\subsubsection{Experiments on datasets with an observational white noise or dynam- ical noise}

Next, we compared univariate and multivariate predictions with datasets that contain observational white noise or dynamical noise. We added observational noise of $10 \%$ and dynamical noise whose $\sigma_{i}$ is $1 \%$ of true standard deviation value. For datasets (ii) and (iii), both of methods provide the prediction of $x_{4}$. The univariate prediction is generated from $x_{4}$, and the 
multivariate prediction is generated from $x_{3}, x_{4}$, and $x_{5}$. For dataset (v), both of methods provide the prediction of $y_{1,1}$. The univariate prediction is generated from $y_{1,1}$, and the multivariate prediction is generated from $x_{8}$, $y_{1,1}$, and $y_{1,2}$.

Figure 2 presents the prediction results for (ii) and (v). Comparing these with the results for (i) and (iv), we observe that the absolute error of the univariate method is much larger in the present case. In addition, the error bars of the univariate prediction give larger values than those for datasets (i) and (iv). These characteristics indicate that the multivariate method is more suitable than the univariate method for a dataset that contains observational noise, and that the multivariate prediction is less strongly affected by observational white noise. This result indicates that our results are valid even if our observations are subjected to observational noise.

Figure 3 presents the prediction results for (iii). Comparing with the result of dataset (i), both of prediction results become worth, but they have the same characteristics as datasets (i) and (ii). The univariate prediction is more useful than any other predictions for short-term predictions, and the multivariate prediction is more accurate than any other predictions for a longer-term prediction. These results indicate that the univariate prediction and the multivariate prediction are useful even if the dynamical system contains dynamical noise.

\subsection{Relationship between observational noise and prediction error}

We inspected the prediction error of dataset (ii) as the observational noise was changed. The graph at the upper left of Fig. 4 presents the result of a onestep prediction in the future for (ii) when the observational noise is changed. Furthermore, the graph at the upper right presents the prediction for five steps ahead, and the graph at the lower presents that for 10 steps ahead. The results indicate that the univariate prediction performs better than the multivariate prediction for a dataset with a small observational noise level over several steps. However, the result for the univariate prediction with a large observational noise level is as accurate as that for the multivariate prediction. In addition, as the prediction steps become longer, the accuracy of the univariate prediction deteriorates. In the presence of a large observational noise, the multivariate prediction gives a superior performance as compared to the univariate prediction. 

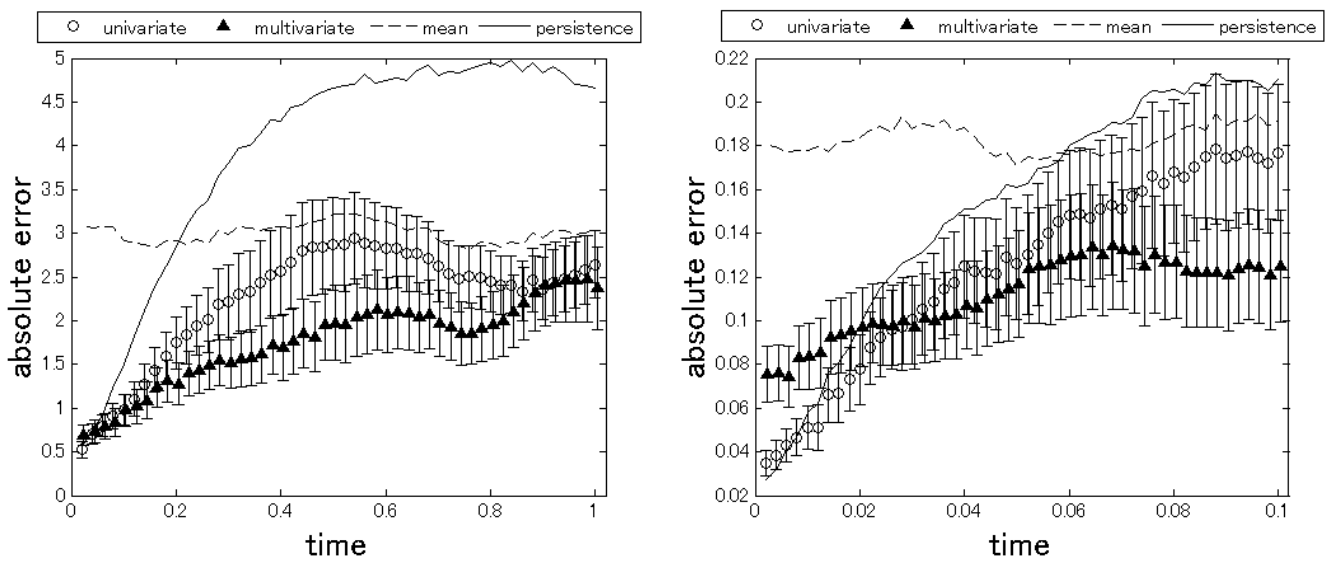

Figure 2: Prediction results for the time series data for the Lorenz 96 I (left) and II (right) model containing 10\% observational noise. The methods applied are univariate and multivariate predictions. An average was taken over 100 runs for each step. We fixed $d=30$. The number of neighboring points is $K=10$. The scale of the error bar is $20 \%$ of the standard deviation.

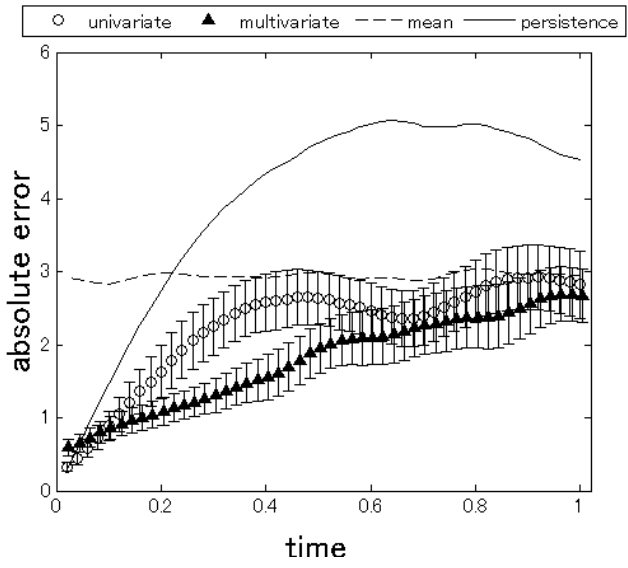

Figure 3: Prediction results for the time series data for the Lorenz 96 I model containing $1 \%$ dynamical noise. The methods applied are univariate and multivariate predictions. An average was taken over 100 runs for each step. We fixed $d=30$. The number of neighboring points is $K=10$. The scale of the error bar is $20 \%$ of the standard deviation. 

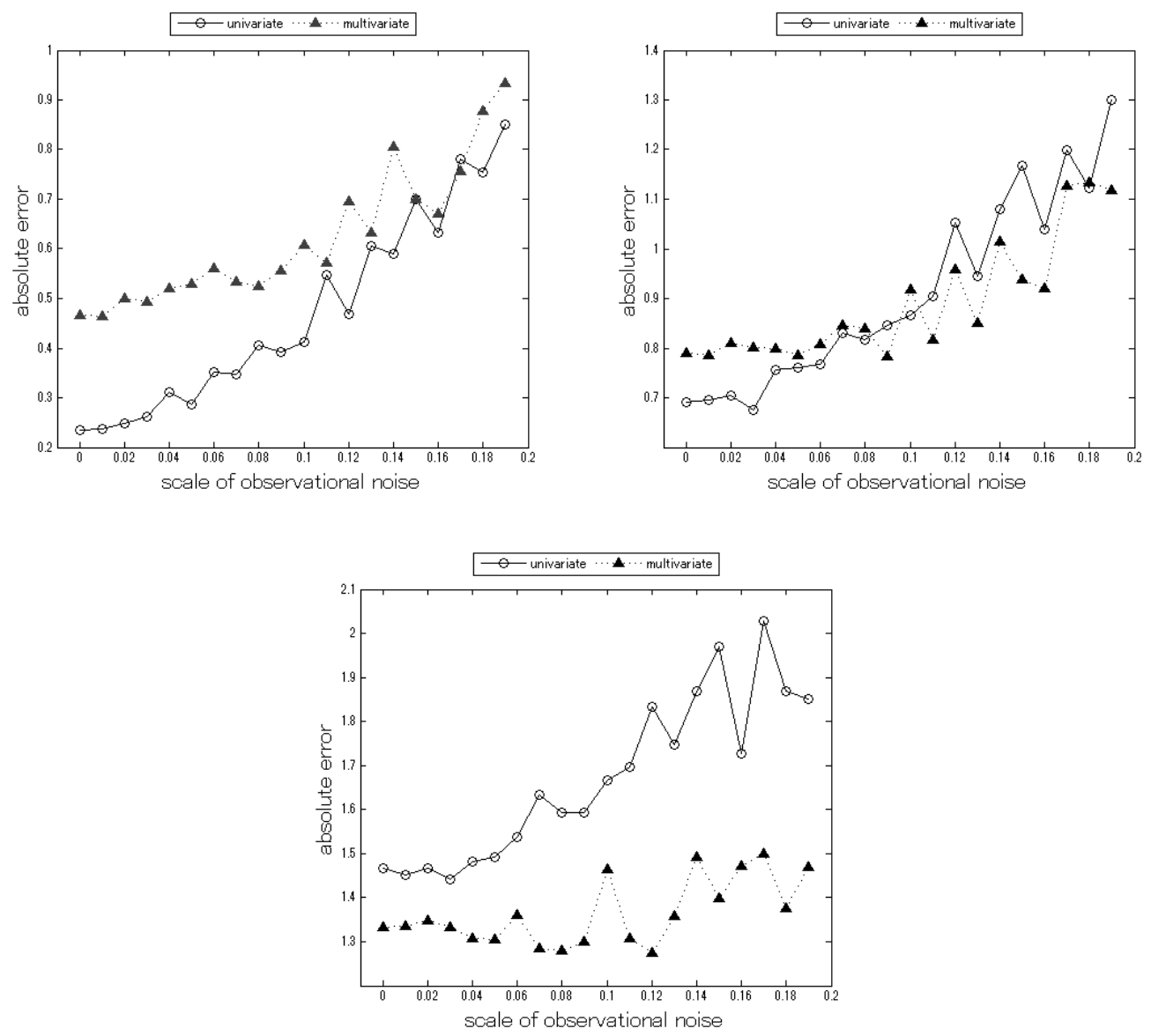

Figure 4: The result for 1-step (upper left), 5-step (upper right), and 10-step (lower) predictions for a time series constructed from the Lorenz 96 I model containing a changing observational noise. We set $d=30$. 


\subsection{Observational data for solar irradiance}

In modern times, the prediction of hourly solar irradiance represents an important problem because solar power production from photovoltaic systems is unstable and we should consider how solar energy can be effectively used. The prediction of solar irradiance has been investigated using various methods. In particular, it has been studied in $[8,15]$ based on a linear approach. In this section, we discuss a numerical experiment for time series data representing solar irradiance that was observed at 10-min intervals at three partially correlated sites. We use the measurements observed at Hiroshima, Takamatsu, and Shimonoseki for our predictions. The results are calculated based on data provided by the Japan Meteorological Agency. The time period represented by the dataset is from January 1, 2010 to December 31, 2010. Table 1 presents the distances between 2 points of 3 observatories, and Table 2 presents the correlation coefficients. These results indicate that solar irradiances of 3 points are correlated with each other, and the greater the distance between 2 points becomes, the smaller the correlation coefficient becomes. These results are consistent with the argument presented by Perez [4]. The relation between correlation coefficients and distances indicates that a multivariate prediction method could be better than a univariate prediction for predicting solar irradiance if we can choose an appropriate setting.

Table 1: Distances between two points $(\mathrm{km})$.

\begin{tabular}{|c||c|c|c|}
\hline & Hiroshima & Takamatsu & Shimonoseki \\
\hline \hline Hiroshima & $*$ & 161.67 & 175.71 \\
\hline Takamatsu & 161.67 & $*$ & 328.67 \\
\hline Shimonoseki & 175.71 & 328.67 & $*$ \\
\hline
\end{tabular}

Table 2: Correlation coefficients between two points.

\begin{tabular}{|c||c|c|c|}
\hline & Hiroshima & Takamatsu & Shimonoseki \\
\hline \hline Hiroshima & $*$ & 0.8802 & 0.8720 \\
\hline Takamatsu & 0.8802 & $*$ & 0.8430 \\
\hline Shimonoseki & 0.8720 & 0.8430 & $*$ \\
\hline
\end{tabular}




\subsubsection{Time series prediction for solar irradiance}

Figure 5 presents the results of a numerical experiment that applies univariate and multivariate predictions for the daytime only. For comparison, we calculated the averages values over 10-min intervals. For a lower number of steps, univariate prediction performed better than multivariate prediction. This relationship was reversed as the numbers of steps became larger. The results are compatible with those of Figs. 1 and 2. According to Boland [8], there is no significant correlation between the residual series for the data representing each 10-min period. He studied cross correlations between different sites, and his research is based on a linear method. His result is consistent with the result of our experiment, while our experiment is based on a nonlinear method. Therefore, we conclude that multivariate model-free prediction is inferior to univariate model-free prediction for short-term predictions. The value of the absolute error of prediction became smaller as the number of prediction steps became larger. However, the cause of this result is that a prediction for a larger number of steps is simpler because the majority of the prediction results for a larger number of steps belong to the nighttime. The graph at the right of Fig. 5 presents the results for a numerical experiment that applies univariate and multivariate predictions for all times. For comparison, the graph at the left of Fig. 5 presents the results of daytime. These results indicate that univariate time series prediction is superior to multivariate prediction for short-term predictions, while multivariate prediction performs better for longer-term predictions. 

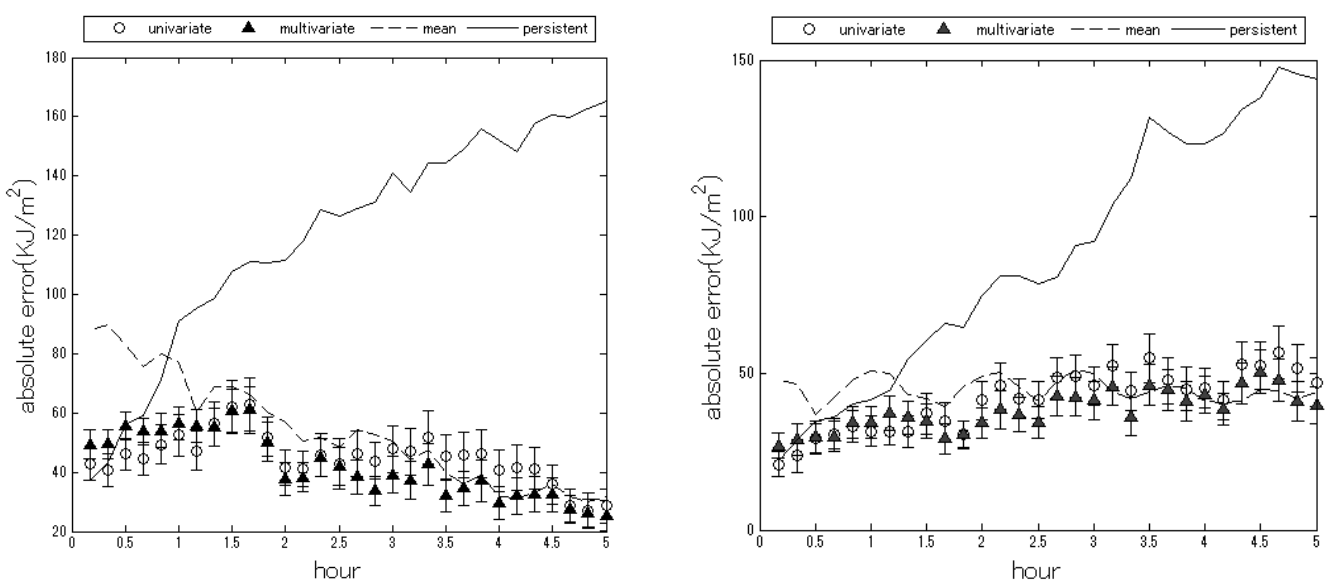

Figure 5: Prediction error for solar irradiance at daytime (left) and all times (right). The methods applied are univariate and multivariate prediction. An average was taken over 50 runs for each step. The scale of the error bar is $20 \%$ of the standard deviation. 


\section{Discussion}

\subsection{Direct prediction vs iterative prediction}

We compared direct and iterative prediction methods. Figure 6 presents the prediction results for dataset (i), for univariate and multivariate predictions, and for direct and iterative ones. For most of prediction, the direct prediction is more accurate than the iterative one. This result means that in this case, the direct prediction is more useful than the iterate prediction when we use the analogue method. The direct prediction seems better than the iterative one because one step prediction for the univariate prediction is not so accurate [16]. Because of these results, we used the direct prediction for numerical experiences.
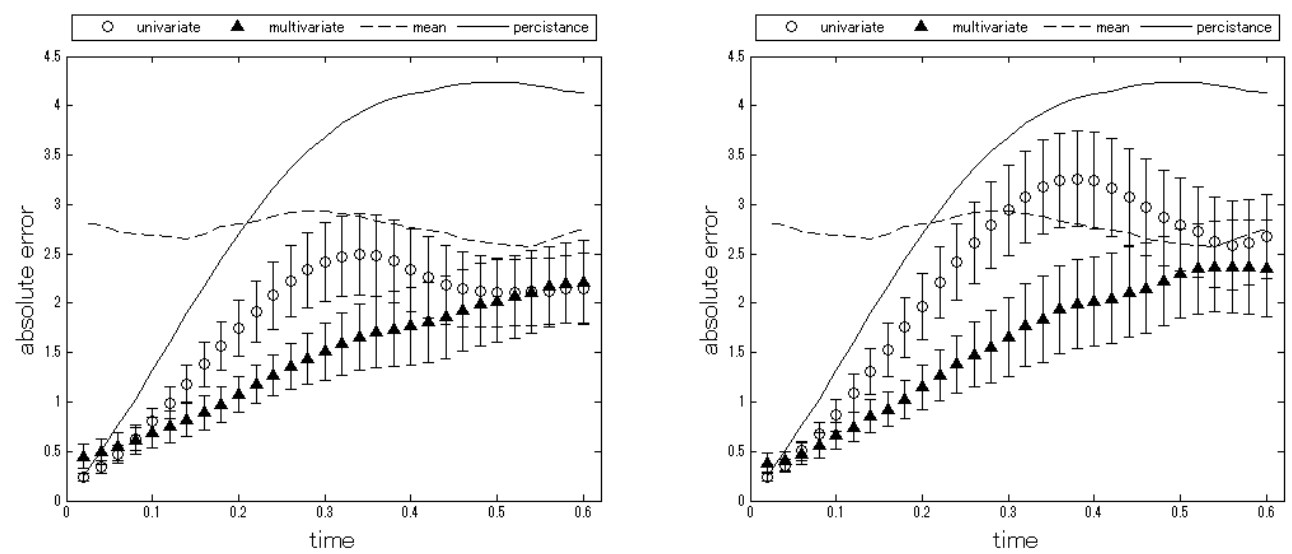

Figure 6: Prediction results for time series data for the Lorenz 96 I model. The methods applied are univariate and multivariate predictions in direct (left) and iterative (right) methods. An average was taken over 100 runs for each step. We fixed $d=30$. The number of neighboring points is $K=10$. The scale of the error bar is $20 \%$ of the standard deviation.

\subsection{Parameter sensitivity}

Furthermore, we investigated the relationships between prediction error and some parameters $d, K$, and $T$ for our prediction.

First, Fig. 7 presents the relationship between the prediction error and the delay dimension. When $d$ is in the range between 1 and 50 , both of the predictions have a robustness of the variation of $d$ except for $d=1$, where the dimension for the delay coordinates is too small and invalid. 
Second, we present, in Fig. 8, relationship between the prediction error and the number of the nearest neighbors. According to the results, both of the predictions are robust in terms of the variation of $K$.

Third, Fig. 9 shows the relationship between the prediction error and the time delay. In this experiment, the time step for integrating differential equations is fixed to $d t=0.0005$ ? in order to investigate the details of $T$. According to the results, the prediction errors for the univariate prediction increase when $T$ increases, while the multivariate prediction is robust when $T$ is varied. These results indicate that the multivariate prediction provides more reliable prediction when the observation interval is long, and that our finding is valid when $T$ is small.
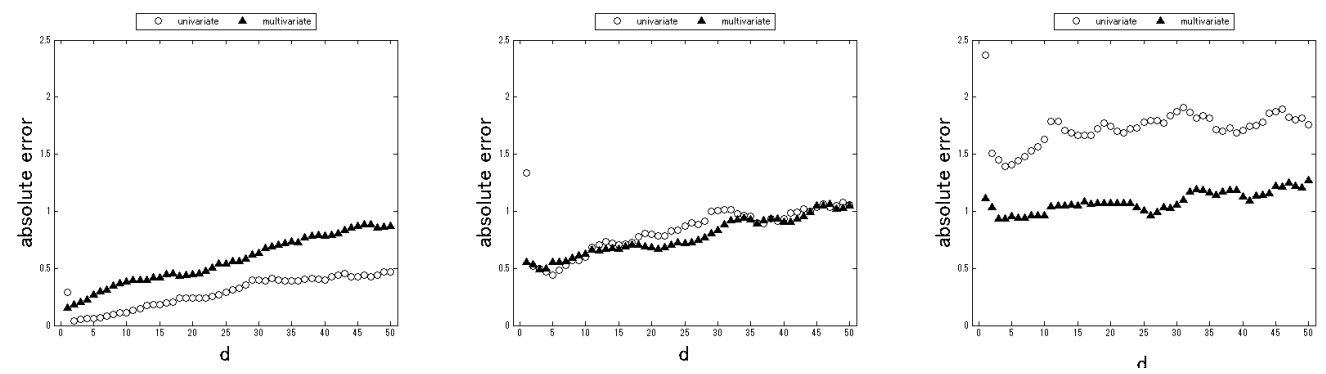

Figure 7: The relationship between the prediction error and the embedding dimension $d$ for Lorenz96 I model. Each panel corresponds to 1 steps ahead (0.02) prediction (left), 5 steps ahead (0.1) prediction (center), and 10 steps ahead (0.2) prediction (right). We set $K=10$ and $T=0.02$.
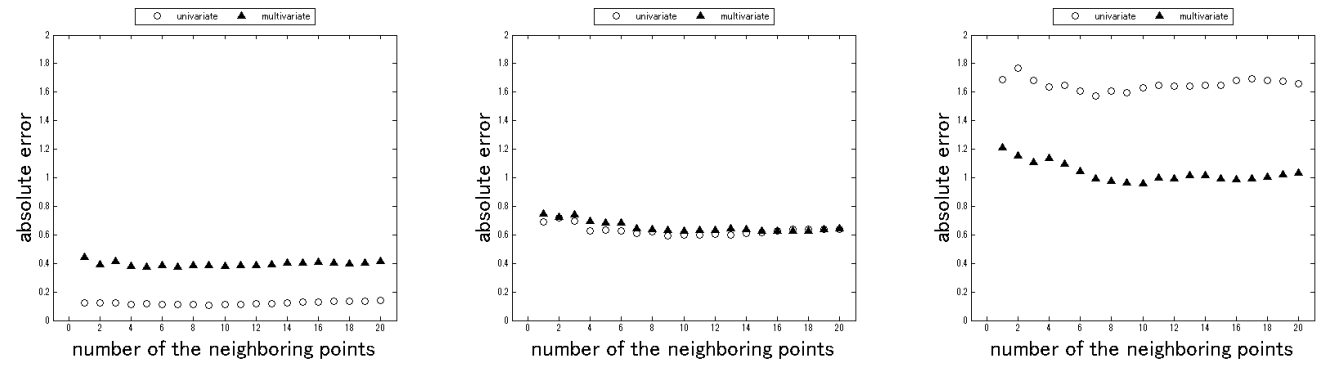

Figure 8: The relationship between the prediction error and the number of the nearest neighbors $K$ for Lorenz 96 I model. Each panel corresponds to 1 steps ahead (0.02) prediction (left), 5 steps ahead (0.1) prediction (center), and 10 steps ahead (0.2) prediction (right). We set $d=30$ and $T=0.02$. 

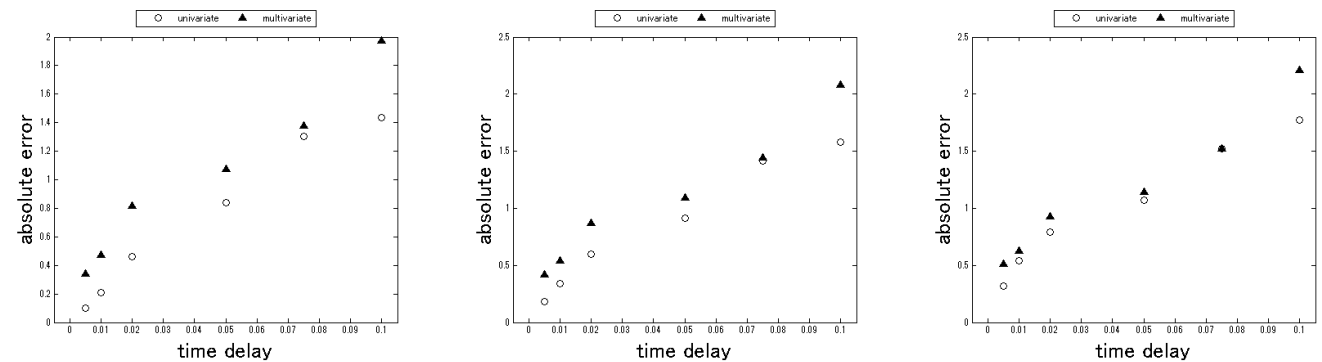

Figure 9: The relationship between the prediction error and the time delay $T$ for Lorenz96 I model. Each panel corresponds to 1 step (0.005) ahead prediction (left), 5 steps ahead (0.025) prediction (center), and 10 steps (0.05) ahead prediction (right). We set $d=30$ and $K=10$.

\subsection{Conclusion}

We compared the performances of univariate prediction and multivariate prediction for artificial data generated from the Lorenz 96 I and II models [6, 11]. In general, the predictions in univariate and multivariate methods are different from each other, so it is difficult to compare these methods under the same conditions. But, in our case, the same approach could be used in evaluating the model-free predictions because the targets on both methods were the same. Further, we used the Euclidean norm in calculating nearest neighbors. We could consider a more fancy way to evaluate the similarity between parts of time series, and we also feel that the Euclidean distance is not the best. But, we kept using the Euclidean distance because it is simple and common.

For short-term predictions, univariate time series prediction is superior to multivariate, while for longer-term predictions or in the presence of large levels of observational noise, multivariate prediction performs better than univariate prediction. These results are consistent with the argument presented by Boland [8], although he focused on covariances between the residuals of univariate predictions (i.e. using a linear method), while our method is nonlinear. We demonstrated the validity of these results for artificial datasets based on the Lorenz 96 models and a real dataset for solar irradiance. The results obtained for the real dataset are compatible with the results for the artificial datasets. Therefore, in order to obtain the best prediction, a method should be chosen depending on the number of prediction steps or the accuracy of the observations. 


\section{Acknowledgements}

We acknowledge the Japan Meteorological Agency for providing the solar irradiance data used in our study. This dataset is commercially available from the Japan Meteorological Business Support Center. This research is supported by Core Research for Evolutional Science and Technology (CREST), Japan Science and Technology Agency (JST).

\section{References}

[1] F. Takens, Detecting strange attractors in turbulence, Lect. Notes Math. 898 (1981) 366-381.

[2] T. Sauer, J. Yorke, and M. Casdagli, Embedology, J. Stat. Phys. 65 (1991) 579-616.

[3] C. Voyant, M. Muselli, C. Paoli, M.-L. Nivet, Optimization of an artificial neural network dedicated to the multivariate forecasting of daily global radiation, Energy 36 (2011) 348-359.

[4] R. Perez, S. Kivalov, J. Schlemmer, K. Hemker, and T. E. Hoff, Shortterm irradiance variability: Preliminary estimation of station pair correlation as a function of distance, Sol. Energy 86 (2012) 2170-2176.

[5] J. Runge, R. V. Donner, and J. Kurths, Optimal model-free prediction from multivariate time series, Physical Review E 91 (2015) 052909.

[6] E. N. Lorenz, Atmospheric predictability as revealed by naturally occurring analogues. J. Atmos. Sci. 26 (1969) 636-646.

[7] H. Kantz, and T. Schreiber, Nonlinear Time Series Analysis, Cambridge University Press, 2004.

[8] J. Boland, Spatial-temporal forecasting of solar radiation, Renew. Energy 75 (2015) 607-616.

[9] G. Gaspari and S. E. Cohn, Construction of correlation functions in two and three dimensions, Q. J. R. Meteorol. Soc. 125 (1999) 723-757.

[10] H. H. Pesaran and Y. Shin, Generalized impulse response analysis in linear multivariate models, Econ. Lett. 58 (1998) 17-29. 
[11] J. A. Hansen and L. A. Smith, The role of operational constraints on selection schemes for supplementary observations, J. Atmos. Sci. 57 (2000) 2859-2871.

[12] L. Cao, A. Mees, and K. Judd, Dynamics from multivariate time series, Physica D 1211(1998) 75-88.

[13] E. R. Deyle and G. Sugihara, Generalized theorems for nonlinear state space reconstruction, PLoS One 6 (2011) e18295.

[14] P. E. Kloeden, and E. Platen, Numerical Solution of Stochastic Differential Equations, Springer, 1992.

[15] P. Bacher, H. Madsen, and H. A. Nielsen, Online short-term solar power forecasting, Sol. Energy 83 (2009) 1772-1783.

[16] K. Judd, and M. Small, Towards long-term prediction, Physica D 136 (2000) 31-44. 